THÉORÈME 16. Supposons gue les fonctions $r(X), h(X)$ et $g(t)$ satisfassent aux hypothèses du théorème 14 et à la condition (42).

Si le système autonome (52) n'a qu'un seul point singulier $X=\Theta$ et s'il satisfait à l'hypothèse de l'unicité des solutions, pour toute solution $X(t)$ du système (43), définie dans un intervalle $\left(t_{0},+\infty\right)$, on $a$.

$$
\lim _{t \rightarrow \infty} X(t)=\Theta \quad \text { et } \quad \lim _{t \rightarrow \infty} X^{\prime}(t)=\Theta .
$$

Travaux. cités

[1] H.A. Antosiowicz, On non linear differen tial equations of the second order with integrable forcing term, J. London Math. Soc. 30 (1955), p. 64-67.

[2] R. Bellman, Stability theory of differential equations, Now York 1953

[3] B. Manfredi, Sulla stcbilità del moto di sistemi a piì gradi di libertà in eon. dizioni non lineari, Boll. Un. Mat. Ital., Serie III, Anno XI (1956), p. 64-71.

[4] L. Markus, Asymptotically aulonomous differential systemss, Contributions to the theory of non-linear oscillations, Vol. III (1956), p. 17.29.

[5] \%. O pial, Sur une équation diffirentiolle non linéaire du second ordro, Ann. Polon. Math. 8 (1960), p. 65-69.

[6] - Sur la dépendance des solutions d'un système d'équations différentielles de leurs seconds membres. Apptication aux systemes presque autonomes, Ann. Polon. Math. 8 (1960), p. 75-89.

[7] P. Santoro, Un oriterio di limitatezza in futuro delle soluzioni di une equhzione differenziale non lineare, Boll. Un. Mat. Ital., Sorio III, Anno XI (1956), p. 432.

[8] G. Sostini, Criterio di stabilità in un problema di Meceunicu non lineare, Rivista di Mat. Univ. Y'arma 2 (1951), p. 303.314.

[9] - Criterio di stabilità in un problema non lineare di meccunica dei sistemi a più gradi di libertà, Rivista di Mat. Univ. Parma 5 (1954), p. 227-232.

[10] T. Ważowski, Systèmes des équations et des inégalités lifférentielles ordinaires anx deuxièmes membres monotones et leur's applications, Ann. Soc. Pol. Math. $23(1950)$, p. $112-166$

Regu par la Réduction le 11.3 .1959
POLONICI MATHEMATICI

VIII (1960)

\section{Propriêtés d'une intégrale de l'équation parabolique dans un domaine non cylindrique}

par A. Prskonek (Warszawa)

1. Introduction. On désigne par $(X, t)$ les points de l'espace-temps, $r=\left(x_{1}, \ldots, x_{n}\right)$ étant un point variable de l'espace euclidien $E^{(n)}$ à $n$ $(n \geqslant 2)$ dimensions, $t$ - la coordonnée du temps.

Dans cet espace-temps nous considérons pour $t \geqslant 0$ un domaine $D$ à $n+1$ dimensions illimité dans la direction de l'axe du temps $t$ (voir [3])

La frontière du domaine $D$ est composée du domaine borné $\Omega_{0}$ à $n$ dimensions, qui est contenu dans le plan $t=0$, et de la surface $s$ à dimensions (c'est-à-dire une variété à $n$ dimensions dans cet espace-temps); $(X, t),(Y, \tau), \ldots$ désignent les points du domaine $D$, et $(P, t)$, $(Q, \tau), \ldots$ - les points de la surface $s$. Pour abréger nous désignerons le point $(P, t)$ (resp. $(Q, \tau))$ par $P_{t}$ (resp. $Q_{\tau}$ ).

Pour un $t_{0}$ fixé nous identifions les points $\left(X, t_{0}\right)$ aux points $X$. Nous désignons la distance de denx points $X, Y$ par $|X Y|$.

Nous appelons $D_{t}$ un domaine borné, partiel du domaine $D$, situé entre les plans $t=0$ et $t=$ const. Nous désignons par $\Omega_{t}$ un domaine borné à $n$ dimensions, situé dans le plan $t=$ const, qui est une portion de la frontière du domaine $D_{t}$.

Nous appelons $s_{t}$ la portion fermée de la surface $s$, située entre les plans $t=0$ et $t=$ const.

Enfin, désignons par $S_{t}$ la variété à $n-1$ dimensions, formée par l'intersection de la surface $s$ et du plan $t=$ const.

Soit $T$ une constante positive, arbitraire, finie. Considérons le domaine $D_{T}$ et supposons que la surface $s_{T}$ possède, suivant la terminologie de J. Hadamard, une orientation du temps (voir [3]) relative à l'équation aux dérivées partielles du type parabolique:

(1) $\quad H[\varkappa(X, t)]$

$$
=\sum_{i, j=1}^{n} a_{i j}(X, t) \frac{\partial^{2} u}{\partial x_{i} \partial x_{j}}+\sum_{k=1}^{n} b_{k}(X, t) \frac{\partial u}{\partial x_{k}}+c(X, t) u-\frac{\partial u}{\partial t}=0
$$

où les coefficients $a_{i j}(X, t), b_{k}(X, t), c(X, t)$ sont continus dans le produit 
cartésien $Q \times\langle 0, T\rangle$, contenant le domaine $D_{q}$ dans son intérieur, et vérifient la condition de Hölder:

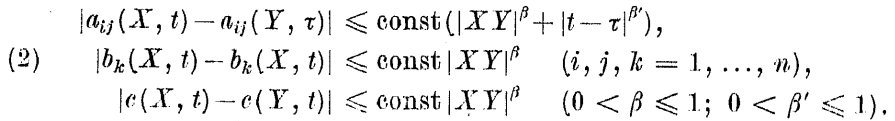

À l'aide de la solution fondamentale (voir [1]) de l'équation (1) on pent définir les intégrales de cette équation (voir [2]), qu'on appolle potentiels généralisés relativement à cette équation, ot qui sonti unalogues aux potentiels relatifs à l'équation du type elliptique.

Récemment W. Pogorzelski (voir [1], [2]) a étudié plusiemrs propriétés de ces intégrales dans le domaine cylindrique $\Omega \times\langle 0, T\rangle$.

Dans ce travail nous démontrerons quelques propriétés d'une intégrale dans le domaine non eylindrique $D_{T}$. On appelle cette intégrale potentiel généralisé de simple couche relatif à l'équation parabolique (1).

2. Propriétés du potentiel de simple couche. Admettons que la surface $s_{T}$ vérifie les conditions suivantes, dites conditions de Liapounoff:

I. Il existe un plan tangent en tout point de la surface $s_{q}$.

II. L'angle $\left(N_{P_{t}}, N_{Q_{\tau}}\right)$ entre deux normales aux points arbitraires $P_{t}$, $Q_{\tau}$ de la surface $\xi_{T}$ vérifie l'inégalité

$$
\left(N_{P_{\ell}}, N_{Q_{\tau}}\right) \leqslant \operatorname{const}\left(|P Q|^{\alpha}+|t-\tau|^{\alpha}\right),
$$

$a$ étant une constante positive, non supérieure à l'unité.

III. Il existe un nombre positif $d$ tel que la sphère $K$ de centre all point arbitraire $P_{t}$ de la surface $s_{T}$ et de rayon $d$, découpe une portion $s_{T}^{K}$ de cétte surface, située à l'intérieữ de la sphère $K$, dont la projection orthogonale sur le plan tangent au point $P_{t}$ est un ensemble de points qui correspondent dans cette projection d'une façon biunivoque aux points de la portion $s_{T}^{K}$.

DéfrnItron. Nous appelons potentiel de simple couche, relatif à l'écquation parabolique (1), l'intégrale de surface $s_{T}$ suivante( $\left.{ }^{1}\right)$ :

$$
\begin{aligned}
U(X, t) & =\boldsymbol{S S}_{s_{t}} \Gamma(X, t ; Q, \tau) \varphi(Q, \tau) d s_{Q_{\tau}} \\
& =\int_{0}^{t} \int_{S_{\tau}} \int_{\tau} \Gamma(X, t ; Q, \tau) \frac{\varphi(Q, \tau)}{\sin \left(N_{Q_{\tau}}, t\right)} d S_{Q} d \tau,
\end{aligned}
$$

(1) On désigne par SS, suivant la notation de J. Hadamard, l'intégrale des surface à $n$ dimensions dans l'espace-temps à $n+1$ dimensions. Nous conservons lo signe d'intégralo double pour l'intégrale de surface à $n-1$ dimensions dans l'espace euclidien $E^{(n)}$ à $n$ dimensions. où : $Y(X, t ; Q, \tau)$ ost la solution fondamentale de l'équation $(1) ; \varphi(Q, \tau)$ est une fonction, dite densité de simple couche, définie sur la surface $s_{T}$, où elle est bornée et intégrable; $d s_{Q_{\tau}}$ désigne l'élément d'aire au point $Q_{\tau}$ de la surface $s_{r} ; d S_{Q}$ - l'élément d'aire an point $Q$ de la variété $s_{\tau}$; $\left(N_{Q_{\tau}}, t\right)$ - l'angle que fait avec l'axe $t$ la normale intérieure $N_{Q_{\tau}}$ à la surface $s_{T}$ au point $Q_{\tau}$.

La fonction (3) vérifie l'équation parabolique (1) en tout point non situé sur la surface $s_{T}$. On peut aussi définir la valeur de la fonction (3) en tout point $P_{t}$ de la surface $s_{T}$ par l'intégrale singulière:

$$
V(P, t)=\lim _{\varepsilon \rightarrow 0} \int_{0}^{t-\varepsilon} \int_{S_{\tau}} \int_{\tau} I(P, t ; Q, \tau) \frac{\varphi(Q, \tau)}{\sin \left(N_{Q_{\tau}}, t\right)} d S_{Q} d \tau
$$

puisque la solution fondamentale $\Gamma(P, t ; Q, \tau)$ admet une singularité pour $(Q, \tau)=(P, t)$.

Pour démontrer l'existence de cette intégrale, considérons au point $P_{t}=(P, t)$ de la surface $s_{r}$ le système de $n+1$ axes rectangulaires $P \xi_{1}, \ldots, \xi_{n}, t$ (admettant le point $P_{t}=(P, t)$ comme origine). L'axe $P \xi_{n}$ de ce système est la normale intérieure $n_{P}$ à le variété $S_{t}$ au point $P$, et les axes $P \xi_{1}, P \xi_{2}, \ldots, P \xi_{n-1}$ sont situés dans le plan tangent à cette variété $S_{t}$ au point $P$.

D'après les conditions de Liapounoff (voir p. 126, I, II, III) il existe une portion suffisamment petite de cette surface $s_{T}$ contenant le point $P_{t}=(P, t)$ telle que la projection $Q_{\tau}^{\prime}=\left(Q^{\prime}, \tau\right)$ de chaque point $Q_{\tau}=(Q, \tau)$ de cette portion sur le plan tangent au point $P_{t}=(P, t)$ ne correspond qu'à un point $Q_{\tau}=(Q, \tau)$ de cette portion, et que la coordonnée $q_{n}$ du point $Q_{\tau}=(Q, \tau)$ est une fonction des coordonnées $q_{1}, \ldots, q_{n-1}$ et $\tau$, c'est-à-dire

$$
q_{n}=g\left(q_{1}, \ldots, q_{n-1}, \tau\right),
$$

où la fonction $g(\ldots)$ possède des dérivées premières remplissant la condition de Hölder.

Pour les points $(Q, \tau)$ d'une portion suffisamment petite de la surface $8_{x}$, contenant le point $(P, t)$, on peut déduire, d'après la formule $(5)$, l'égalité

$$
\begin{aligned}
& \text { (6) }|P Q|^{2}=\left|P Q^{\prime}\right|^{2}\left(1+\left(\sum_{i=1}^{n-1} \frac{\partial g\left(Q^{*}, \tau^{*}\right)}{\partial q_{i}} a_{i}\right)^{2}\right)- \\
& -2\left|P Q^{\prime}\right|\left(\sum_{i=1}^{n-1} \frac{\partial g\left(Q^{*}, \tau^{*}\right)}{\partial q_{i}} a_{i}\right) \frac{\partial g\left(Q^{*}, \tau^{*}\right)}{\partial \tau}(t-\tau)+\left(\frac{\partial g\left(Q^{*}, \tau^{*}\right)}{\partial \tau}\right)^{2}(t-\tau)^{2}
\end{aligned}
$$

où: $Q^{\prime}$ est la projection du point $Q$ sur le plan tangent au point $P$ de la 
variété $S_{t} ; \alpha_{i}-$ les cosinus directeurs du vectem $\overrightarrow{P Q^{\prime}}$ pour $i=1, \ldots, n-1$. $Q^{*}$ - un point à l'intérieur du segment $P Q^{\prime} ; \tau^{*}$ - me valeur du ti(mps à l'intérieur de l'intervalle $(\tau, t)$.

D'après la formule (6), en tenant compte de la limitation connue (voir [1], [2]) à singularités séparées de la solution fondamentale $\Gamma(P, t ; Q, \tau)$, et en raison de l'orientation du temps de la surface sq, on obtient une inégalité de la formo

$$
\left|\Gamma(P, t ; Q, \tau) \frac{\varphi(Q, \tau)}{\sin \left(N_{Q_{\tau}}, t\right)}\right| \leqslant \frac{\text { const } M_{p}}{(t-\tau)^{\prime \prime}\left|P Q^{\prime}\right|^{n-2 \mu}},
$$

où $M_{p}=\sup _{(Q, \tau) \in S_{T}}|\varphi(Q, \tau)|, \mu=$ constante positive, arbitraire; const: =- constante positive, qui ne dépend que de la surface $s_{T}$, du domaine $I_{T}$, des coefficients de l'équation (1) et de la constante $\mu$.

En choisissant la constante positive $\mu$ arbitrairement à l'intérieur de l'intervalle $\left(\frac{1}{2}, 1\right)$ nous voyons que la fonction sous le signe de l'intégrale singulière (4) admet une singularité faible, si $\tau \rightarrow t, Q \rightarrow P$, et par conséquent cette intégrale est absolument et uniformément convergente. On peut démontrer par la méthode classique de la théorio du potentiel que le potentiel (3) vérifie la relation suivante:

$$
\lim _{X \rightarrow P} U(X, t)=U(P, t) .
$$

D'après l'inégalité (7), le potentiel (3) admet la limitation

$$
|U(X, t)| \leqslant \text { const } M_{\varphi} t^{1-\mu} \text {. }
$$

Cette inégalité nous apprend que le potentiel (3) tend uniformément vers zéro, si $t \rightarrow 0$.

Remarquons encore que le potentiel (3) est deux fois dérivable on tout point non situé sur la surface $s_{T}$, sous la seule supposition. que la fonction $\varphi(Q, \tau)$ soit bornée et intégrable.

Les dérivées premières spatiales de la solution fondamentale $\Gamma(P, t ; Q, \tau)$ admettent une limitation à singularité forte relativement à l'intégrale de surface. Done nous ne pouvons pas affirmer, même si la fonction $\varphi(Q, \tau)$ était continue, que les dérivées du potentiel (3) ont dos valeurs limites, si le point intérieur $(X, t)$ tend vers un point $(P, t)$ de la surface $s_{T}$. Cependant une certaine combinaison linéaire do ces dérivées, dite - d'après $J$. d'Adhémar - dérivée transversale de la fonction $U(X, t)$ relativement au point $P_{t}=(P, t)$ de la surface $s_{T}$, donnéo par la relation

$$
\frac{d U(X, t)}{d T_{P_{t}}}=\sum_{i, j=1}^{n} a_{i j}(X, t) \frac{\partial U(X, t)}{\partial x_{i}} \cos \left(N_{P_{t},}, x_{j}\right),
$$

a une limite déterminéc, si $\boldsymbol{X} \rightarrow P$; $\left(N_{P_{t}}, x_{j}\right)$ désigne l'angle, que fait avec l'axe $x_{j}$ la normale intérieure $N_{P_{t}}$ à la surface $s_{T}$ au point $(P, t)$. Nous pouvons écrire la relation (10)

$$
\frac{d U(X, t)}{d T_{P_{t}}}=\sum_{i, j=1}^{n} s_{i j}(X, t) \frac{\partial U(X, t)}{\partial x_{i}} \cos \left(n_{P}, x_{j}\right) \sin \left(N_{P_{t}}, t\right)
$$

et désigner pour abréger

$$
\frac{d U(X, t)}{d T_{P_{t}}}=\frac{d U(X, t)}{d v_{P}} \sin \left(N_{P_{t}}, t\right)
$$

D'après la relation (10) et les notations (11), (12) on peut exprimer la dérivée transversale du potentiel (3) sous la forme suivante:

(13) $\frac{d U(X, t)}{d T_{P_{t}}}=\int_{0}^{t} \int_{S_{\tau}} \int_{\frac{d}{2}(X, t ; Q, \tau)} \cdot \frac{\varphi(Q, \tau)}{d \nu_{P}} d S_{Q} d \tau \cdot \sin \left(N_{P_{t}}, t\right)$.

Écrivons la dérivée transversale de la solution fondamentale $\Gamma(X, t ; Q ; \tau)$ sous forme (voir [2]) d'une somme:

$$
\begin{aligned}
& \text { (14) } \frac{d \Gamma(X, t ; Q, \tau)}{d T_{P_{t}}} \\
& =\left\{-\frac{|X Q| \cos \left(\overrightarrow{Q X}, n_{P}\right) \exp \left[-\frac{\theta^{(Q, \tau)}(X, Q)}{4(t-\tau)}\right]}{2(t-\tau)^{n / 2+1}}+\tilde{F}(X, t ; Q, \tau)\right\} \sin \left(N_{P_{t}}, t\right),
\end{aligned}
$$

où $\Theta^{(Q, \tau)}(X, Q)=\sum_{i, j=1}^{n} a^{i j}(Q, \tau)\left(x_{i}-q_{i}\right)\left(x_{j}-q_{j}\right), \quad a^{i j}(Q, \tau)$ désigne les éléments de la matrice inverse de la matrice $\left[a_{i j}(Q, \tau)\right]$.

D'après cette formule, nous pouvons écrire la dérivée transversale du potentiel $U(X, t)$ sous forme d'une somme d'intégrales:

oì

$$
\frac{d U(X, t)}{d T_{P_{t}}}=J(X, t)+\tilde{J}(X, t)
$$

$$
\begin{gathered}
J(X, t)=-\int_{0}^{t} \int_{S_{\tau}^{*}} \frac{|X Q| \cos \left(\overrightarrow{Q X}, n_{P}\right) \exp \left[-\Theta^{(Q, \tau)}(X, Q) / 4(t-\tau)\right]}{2(t-\tau)^{n / 2+1}} \times \\
\times \frac{\varphi(Q, \tau)}{\sin \left(N_{Q_{\tau}}, t\right)} d S_{Q} d \tau \cdot \sin \left(N_{P_{t}}, t\right), \\
\tilde{J}(X, t)=\int_{0}^{t} \int_{S_{\tau}} \int_{\tilde{\tau}} \tilde{F}(X, t ; Q, \tau) \frac{\varphi(Q, \tau)}{\sin \left(N_{Q_{\tau}}, t\right)} d S_{Q} d \tau \cdot \sin \left(N_{P_{t}}, t\right) .
\end{gathered}
$$

Annales Polonici Mathematici virr 
On peut aussi définir la valeur de l'intégrale (13) en tout point $(P, t)$ de la surface $s_{T}$ par l'intégrale singulière:

(16) $\quad V(P, t)=\lim _{Q \rightarrow 0} \int_{0}^{t-\varepsilon} \int_{S_{\tau}} \int_{\frac{d \Gamma(P, t ; Q, \tau)}{d \nu_{P}}} \frac{\varphi(\dot{Q}, \tau)}{\sin \left(N_{Q_{\tau}}, t\right)} d S_{Q} d \tau \cdot \sin \left(N_{P_{t}}, t\right)$.

Décomposons cette intégrale singulière, d'après la formule (14), en somme de deux intégrales:

$$
V(P, t)=v(P, t)+\tilde{v}(P, t),
$$

où a

$\left(17^{1}\right) \quad v(P, t)=-\lim _{\varepsilon \rightarrow 0} \int_{0}^{t-s} \int_{S_{\tau}} \int_{|P Q| \cos \left(\overrightarrow{Q P}, n_{P}\right) \exp \left[-\Theta^{(Q, \tau)}(P, Q) / 4(t-\tau)\right]}^{2(t-\tau)^{n / 2+1}} \times$

$$
\times \frac{\varphi(Q, \tau)}{\sin \left(N_{Q_{\tau}}, t\right)} d S_{Q} d \tau \cdot \sin \left(N_{P_{t}}, t\right),
$$

(17 $) \quad \tilde{v}(P, t)=\lim _{Q \rightarrow 0} \int_{0}^{t-\varepsilon} \int_{S_{\tau}} \int_{\tilde{F}} \tilde{F}(P, t ; Q, \tau) \frac{\varphi(Q, \tau)}{\sin \left(N_{Q_{\tau}}, t\right)} d S_{Q} d \tau \cdot \sin \left(N_{P_{\ell}}, t\right)$.

Passons à l'étude de intégrale (13). Dans ce but nous démontrorons d'abord les trois lemmes suivants:

LEMME 1. Si la densité $\varphi(Q, \tau)$ est une fonction bornée et intégrable sur la surface $s_{T}$, alors l'intégrale singulière $\left(17^{2}\right)$ est absolument et uniformément convergente et la fonction $\left(16^{2}\right)$ tend uniformément vers une limite déterminée par cette intégrale singulière:

$$
\lim _{X \rightarrow P} \tilde{J}(X, t)=\tilde{v}(P, t),
$$

si le point $(X, t)$ tend vers le point $(P, t)$.

Démonstration. D'après les travaux [1], [2] et la formule (6), la fonction $\tilde{F}(P, t ; Q, \tau)$ rérifie l'inégalité

$$
|\tilde{F}(P, t ; Q, \tau)| \leqslant \frac{\text { const }}{(t-\tau)^{\mu}\left|P Q^{\prime}\right|^{n+1-2 \mu-\beta_{0}}},
$$

où $\beta_{0}=\min \left(\beta, 2 \beta^{\prime}\right)$.

Done, en fixant l'exposant $\mu$ à l'intérieur de l'intervalle $\left(1-\frac{1}{2} \beta_{0}, 1\right)$, nous pouvons établir par un raisonnement classique que l'intégrale singulière $\left(17^{2}\right)$ est absolument et uniformément convergente, en outre la fonction $\left(\mathbf{1 6}^{2}\right)$ tend uniformément, vers la limite déterminée par cette intégrale singulière, c'est-à-dire vérifie l'égalité $(1,8)$.
D'après l'inégalité (19), la fonction $\left(16^{2}\right)$ admet la limitation

$$
|\tilde{J}(X, t)| \leqslant \text { const } M_{\varphi} f^{1-\mu} .
$$

Done cette fonction tend vers zéro, si $t \rightarrow 0$

LEMTME 2. Si la densité $\varphi(Q, \tau)$ est une fonction bornée et intégrable sur la surface $8_{T}$, satisfaisant aux conditions de Liapounoff (voir p. 126, I, II, III), l'intégrale singulière (1,71) est absolument et uniformément convergente.

Démonstration. Désignons par $\pi_{t}$ le plan tangent au point $P_{t}$ à la surface $s_{T}$, et par $\Pi_{t}-$ la variété à $n-1$ dimensions, formée par l'intersection des plans $t=$ const et $\pi_{t}$. Les axes $P \xi_{1}, P \xi_{2}, \ldots, P \xi_{n-1}$ du système de coordonnées $P \xi_{1}, \ldots, \xi_{n}, t$ (voir p. 127) sont contenus dans cette variété $\Pi_{t}$.

Consídérons dans le plan $t=$ const (c'est-à-dire dans l'espace euclidien $E^{(n)}$ à $n$ dimensions) un cylindre $W\left(P, \varrho_{0}\right)$, d'axe $P \xi_{n}$ et de rayon $\varrho_{0}$, qui découpe une portion $\Sigma_{t}^{\theta}$ de la variété $S_{t}$ au voisinage du point $P$; soit en outre une sphère $\Pi_{t}^{0}$ à $n-1$ dimensions - projection de la portion $\Sigma_{t}^{0}-$ de la variété $\Pi_{t}$. Ce cylindre $W\left(P, \varrho_{0}\right)$ découpe dans le plan $\tau=$ const une portion $\Sigma_{\tau}^{0}$ de la variété $S_{\tau}$ et une sphère $\Pi_{\tau}^{0}$ de la variété $\Pi_{\tau}$.

Appelons bande $\sigma_{t}^{0}$ de la surface ${ }^{s_{T}}$ l'ensemble de tous les points $(Q, \tau)$ qui satisfont à la condition

$$
Q \in \Sigma_{\tau}^{0} ; \quad 0 \leqslant \tau \leqslant t .
$$

Pareillement appelons bande $\Pi_{\tau}^{0} \times\langle 0, t\rangle$ du plan $P \xi_{1}, \ldots, \xi_{n-1}, t$ l'ensemble de tous les points $\left(Q^{\prime}, \tau\right)$ qui satisfont'à la condition

$$
Q^{\prime} \in \Pi_{t}^{0}, \quad 0 \leqslant \tau \leqslant t .
$$

Remarquons que la bande $\Pi_{i}^{0} \times\langle 0, t\rangle$ est la projection de la bande $\sigma_{t}^{0}$ sur le plan $P \xi_{1}, \ldots, \xi_{n-1}, t$. Remarquons ensuite que, d'après les conditions de Liapounoff, nous pouvons toujours fixer un rayon $\varrho_{0}$ suffisamment petit, par exemple $\varrho_{0}=d / 3$, pour que les points de la sphère $\Pi_{t}^{0}$ correspondent, d'une facon biunivoque, aux points de la portion $\Sigma_{t}^{0}$.

Décomposons l'intégrale singulière $\left(\mathbf{1 7}^{1}\right)$ en deux parties

$$
v(P, t)=v^{o_{t}^{0}}(P, t)+v^{v_{t}-\sigma_{i}^{0}}(P, t)
$$

étendues à la bande $\sigma_{t}^{0}$ et à la surface extérieure $s_{t}-\sigma_{t}^{0}$. Le point $(P, t)$ étant extérieur au domaine d'intégration $s_{t}-\sigma_{i}^{0}$, le second terme de la somme est continu au point $(\boldsymbol{P}, t)$.

Pour étudier la première des intégrales (23), remarquons que tout 
point $Q$ de la portion $\Sigma_{\tau}^{0}$ (la différence $t-\tau$ étant suffisamment petite) satisfait aux inégalités connues de Liapounoff:

$$
\text { (24) } \quad|Q \tilde{Q}| \leqslant \text { const }|\tilde{P} Q|^{1+a}, \quad|\tilde{P} Q| \leqslant 2|\tilde{P} \tilde{Q}|, \quad \cos \left(n_{\tilde{P}}, n_{Q}\right) \geqslant \frac{1}{2}
$$

par rapport au système d'axes rectangulaires $\tilde{P}_{\eta_{1}}, \ldots, \eta_{n}$, l'axe $\tilde{P}_{\eta_{n}}$ étant la normale intérieưe à la variété $S_{r}$ au point $\tilde{P}$, qui est intersection do l'axe $P \xi_{n}$ et de cette variété $S_{\tau}$. Le point $\tilde{Q}$ est la projection orthogonale du point $Q$ sur le plan $\tilde{P}_{\eta_{1}}, \ldots, \eta_{n-1}$ (tangent au point $\tilde{P}$ à la variété $\mathbb{S}_{\tau}$ ). Nous avons done l'inégalité

$$
\text { (25) } \quad|P Q| \cos \left(\overrightarrow{Q P}, n_{P}\right)|\leqslant| P \tilde{P}|+| Q \tilde{Q}\left|\cos \left(n_{P}, n_{\tilde{P}}\right)+\right| \tilde{P} \tilde{Q} \mid \sin \left(n_{P}, n_{\tilde{P}}\right) \text {. }
$$

En profitant de la formule (6) et des inégalités

$$
|\tilde{P} Q| \leqslant|\tilde{P} \boldsymbol{P}|+|P Q|, \quad \sin \left(n_{P}, n_{\tilde{P}}\right) \leqslant \mathrm{const}(t-\boldsymbol{\tau})^{a},
$$

nous pouvons écrire

$$
\text { (27) } \begin{aligned}
& || P Q\left|\cos \left(\overrightarrow{Q P}, n_{P}\right)\right| \\
& \leqslant \operatorname{const}\left\{(t-\tau)\left[\left(1+2|P Q|^{a}\right)+2^{2}(t-\tau)^{a}\right]+|P Q|(t-\tau)+|P Q|^{1+a}\right\} .
\end{aligned}
$$

D'après la formule (6) et d'après l'inégalité (27), la fonction sous la signe de l'intégrale singulière $\left(17^{1}\right)$ vérifie l'inégalité

$$
\text { (28) } \begin{aligned}
\left|-\frac{P Q \cos \left(\overrightarrow{Q P}, n_{P}\right) \exp \left[-\Theta^{(Q, \tau)}(P, Q) / 4(t-\tau)\right]}{2(t-\tau)^{n / 2+1}} \cdot \frac{\varphi(Q, \tau)}{\sin \left(N_{Q_{\tau}}, t\right)}\right| \\
\leqslant \frac{\operatorname{const} M_{\varphi}}{(t-\tau)^{\mu}\left|P Q^{\prime}\right|^{n+1-2 \mu-\alpha}} .
\end{aligned}
$$

Donc, en fixant l'exposant $\mu$ à l'intérieur de l'intervalle $\left(1-\frac{1}{2} \alpha, 1\right)$, nous pouvons affirmer que l'intégrale singulière $v^{v^{0}}(P, t)$ est absolument et uniformément cenvergente. On peut démontrer, par un raisonnement classique, que l'intégrale singulière $\left(17^{1}\right)$ admet la limitation

$$
|U(P, t)| \leqslant \text { const } M_{\varphi} t^{1-\mu} \text {. }
$$

D'après cette inégalité, et d'après l'inégalité (20), la fonction $V(P, t)$ tend uniformément vers zéro, si $t \rightarrow 0$.

Lemme 3. Si la densité $\varphi(Q, \tau)$ est une fonction continue et bornée sur la surface $s_{T}$, satisfaisant aux conditions de Liapounoff, la fonction (151) tend uniformément vers la limite

$$
\lim _{\boldsymbol{X} \rightarrow \boldsymbol{P}} J(X, t)=v(\boldsymbol{P}, t)-\frac{(2 \sqrt{\pi})^{n} \varphi(\boldsymbol{P}, t)}{2 \sqrt{\operatorname{det}\left[a^{i j}(\boldsymbol{P}, t)\right]}}
$$

si le point $X$ tend vers un point $P$ de la variété $S_{t}$, où $a^{i j}(P, t)$ désignent les éléments de la matrice inverse de la matrice $\left[a_{i j}(P, t)\right]$ (voir p. 125, équation (1)).

Démonstration. Considérons l'intégrale suivante:

(31) $J^{*}(X, t)$

$=-\int_{0}^{t} \int_{n_{i}^{0}} \frac{\left|X Q^{\prime}\right| \cos \left(\overrightarrow{Q^{\prime} X}, n_{P}\right) \exp \left[-\Theta^{\left(Q^{\prime}, \tau\right)}\left(X, Q^{\prime}\right) / 4(t-\tau)\right]}{2(t-\tau)^{n / 2+1}} \varphi(P, t) d \Pi_{Q^{\prime}} d \tau$, étendue à la bande $\Pi_{t}^{0} \times\langle 0, t\rangle$ du plan $P \xi_{1}, \ldots, \xi_{n-1}, t$.

D'après le travail [2], cette intégrale possède la propriété limite suivante:

$$
\lim _{X \rightarrow P} J^{*}(X, t)=-\frac{(2 \sqrt{\pi})^{n} \varphi(P, t)}{2 \sqrt{\operatorname{det}\left[a^{i j}(P, t)\right]}} .
$$

Pour démontrer la relation (30), étudions la différence

$$
R(X, t)=J(X, t)-J^{*}(X, t) .
$$

Remarquons que, d'après le lemme 2, la fonction $R(X, t)$ vérifie l'égalité.

$$
R(P, t)=v(P, t)
$$

sur la surface $8_{T}$.

Nous démontrerons maintenant que, pour un $t>0$ fixé, la différence (33) est continue au point $(P, t)$ de la surface $\boldsymbol{s}_{T}$, c'est-à-dire que l'on a

$$
\lim _{X \rightarrow P} R(X, t)=R(P, t) \text {. }
$$

Supposons d'abord que le point $X$ soit situé sur la normale intérieure $n_{P}$ au point arbitraire $P$ de la variété $S_{t}$ et considérons un système d'axes rectangulaires $P \xi_{1}, \ldots, \xi_{n}, t$, où l'axe $P \xi_{n}$ est la normale $n_{P}$.

Soit un cylindre $W\left(P, \varrho_{1}\right)$, d'axe $P \xi_{n}$ et de rayon $\varrho_{1}<\varrho_{0}$, qui découpe une portion $\Sigma_{t}^{1}$ de la variété $S_{l}$ au voisináge du point $P$, et une portion $\Sigma_{\tau}^{\mathrm{l}}$ de la variété $S_{\tau}$.

Ce cylindre $W\left(P, \varrho_{1}\right)$ découpe dans le plan tangent au point $P$ une sphère $\Pi_{t}^{l}$ à $n-1$ dimensions - projection de la portion $\Sigma_{t}^{1}$.

Nous appelons bande $\sigma_{t}^{1}$ de la surface $s_{T}$ l'ensemble de tous les points $(Q, \tau)$ qui satisfont à la condition

$$
Q \in \Sigma_{\tau}^{1}, \quad 0 \leqslant \tau \leqslant t .
$$

Remarquons que la bande $\Pi_{t}^{1} \times\langle 0, t\rangle$ est la projection de la bande $\sigma_{t}^{1}$ sur le plan $P \xi_{1}, \ldots, \xi_{n-1}, t$. 
Décomposons l'intégrale $J(X, t)$ en deux parties étendues à la bande $\sigma_{t}^{1}$ et à la surface extérieure $s_{t}-\sigma_{t}^{1}$, en outre décomposons l'intégrale $J^{*}(X, t)$ en deux parties étendues à la bande $\Pi_{t}^{1} \times\langle 0, t\rangle$ et aux bandes extérieures $\left(\Pi_{t}^{0}-\Pi_{t}^{1}\right) \times\langle 0, t\rangle$.

Nous pouvons alors écrire la différence (33) sous forme d'une somme d'intégrales

(37) $\quad R(X, t)$

$=I_{1}(X, t)+I_{2}(X, t)+I_{3}(X, t)+I_{4}(X, t)$

$=-\int_{0}^{t} \int_{n_{t}^{1}} \int_{1}\left[|X Q| \cos \left(\overrightarrow{Q X}, n_{P}\right)-\left|X Q^{\prime}\right| \cos \left(\overrightarrow{Q^{\prime} X}, n_{P}\right)\right] \times$

$$
\times \frac{\exp \left[-\Theta^{(Q, \tau)}(X, Q) / 4(t-\tau)\right]}{2(t-\tau)^{n / 2+1}}+\left|X Q^{\prime}\right| \cos \left(Q^{\prime} X, n_{P}\right) \times
$$$$
\left.\times \frac{\exp \left[-\Theta^{(Q, \tau)}(X, Q) / 4(t-\tau)\right]-\exp \left[-\Theta^{\left(Q^{\prime}, \tau\right)}\left(X, Q^{\prime}\right) / 4(t-\tau)\right]}{2(t-\tau)^{n / 2+1}}\right\} \times
$$

$\times \frac{\varphi(Q, \tau)}{\sin \left(N_{Q_{\tau}}, t\right) \cos \left(n_{Q}, n_{P}\right)} d \Pi_{Q^{\prime}} d \tau \sin \left(N_{P_{t}}, t\right)-$

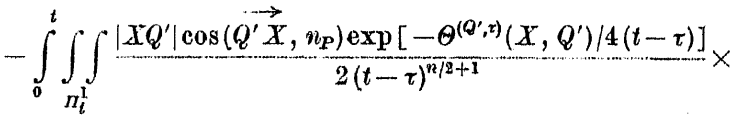

$\times\left[\frac{\varphi(Q, \tau)}{\sin \left(N_{Q_{\tau}}, t\right) \cos \left(n_{Q}, n_{P}\right)}-\frac{\varphi(P, t)}{\sin \left(N_{P_{t}}, t\right)}\right] d I_{Q} d \tau \sin \left(N_{P_{t}}, t\right) \ldots$

$-\int_{\theta}^{t} \iint_{S_{n}-x_{\tau}^{1}} \frac{|X Q| \cos \left(\overrightarrow{Q X}, n_{P}\right) \exp \left[-\Theta^{(Q, \tau)}(X, Q) / 4(t-\tau)\right]}{2(t-\tau)^{n / 2+1}} \times$

$\times \frac{\varphi(Q, \tau)}{\sin \left(N_{Q_{\tau}}, t\right)} d S_{Q} d \tau \sin \left(N_{P_{\ell}}, t\right)-$

$-\int_{\theta}^{t} \int_{n_{i}^{0}-n_{t}^{1}} \int_{\left|X Q^{\prime}\right| \cos \left(\overrightarrow{Q^{\prime} X}, n_{P}\right) \exp \left[-\Theta^{\left(Q^{\prime}, \tau\right)}\left(X, Q^{\prime}\right) / 4(t-\tau)\right]}^{2(t-\tau)^{n / 2+1}} \varphi(P, t) d I I_{Q^{\prime}} d \tau$,

où $Q$ est un point de la variété $S_{\tau}, Q^{\prime}$ est la projection du point $Q$ sur: le plan tangent au point $P$ de la variété $S_{t}$.

Nous désignons par $f(X, t ; Q, \tau)$ la fonction sous le signe de la première intégrale dans la somme (37). Cette fonction vérifie l'inégalité

$$
|f(X, t ; Q, \tau)| \leqslant \frac{\text { const } M_{\varphi}}{(t-\tau)^{\mu}\left|P Q^{\prime}\right|^{n+1-2 \mu-a \beta}},
$$

Les singularités de la limitation (38) sont faibles relativement à cette intégrale, $\mu$ étant choisi à l'intérieur de l'intérvalle $\left(1-\frac{1}{2} \alpha \beta, 1\right)$.

Done cette intégrale tend uniformément vers zéro, si $\varrho_{1} \rightarrow 0$, e'est-à-dire

$$
\lim _{\varrho_{1} \rightarrow 0} I_{1}(X, t)=0
$$

quelle que soit la position du point $X$ sur la normale $n_{P}$.

Remarquons que la deuxième intégrale de la somme (37) est du type (31), et par conséquent, elle possède la propriété limite

$$
\lim _{X \rightarrow \boldsymbol{P}} I_{\mathbf{z}}(X, t)=0
$$

puisque la différence sous le signe de cette intégrale est continue sur la bande $\Pi_{t}^{1} \times\langle 0, t\rangle$ et cette différence est nulle pour $(Q, \tau)=(P, t)$.

La troisième et la quatrième des intégrales de la somme (37) sont continues au point $(P, t)$ de la surface $s_{\boldsymbol{T}}$, e'est-à-dire que l'on a

$$
\begin{gathered}
\lim _{X \rightarrow P} I_{3}(X, t)=I_{3}(P, t), \\
\lim _{X \rightarrow P} I_{4}(X, t)=0 .
\end{gathered}
$$

Étudions done la différence

(43) $\quad R(X, t)-R(P, t)$

$$
=I_{1}(X, t)-I_{1}(P, t)+I_{2}(X, t)-I_{3}(X, t)-I_{3}(P, t)+I_{4}(X, t),
$$

si $X \rightarrow P$.

Remarquons que, d'après la relation (39), les valeurs de la première intégrale sont arbitrairement petites, si l'on prend une valeur $\varrho_{1} \neq 0$ suffisamment petite.

Remarquons ensuite que, d'après les relations (40), (41) et (42), les termes de la somme (43) $I_{2}(X, t), I_{3}(X, t)-I_{3}(P, t)$, et $I_{4}(X, t)$ sont arbitrairement petits, si $|X P|$ est suftisamment petite.

En réunissant les résultats obtenus nous arrivons à la conclusion (30), e'est-à-dire

$$
|R(X, t)-R(P, t)|<\varepsilon, \quad \text { si } \quad|X P|<\eta(\varepsilon)
$$

dans le cas où le point $X$ est situé sur la normale au point $P$.

En appliquant le raisonnement de W. Pogorzelski (voir [2], démonstration du théorème 1) on peut démontrer l'égalité (30) dans lo cas où le point $X$ tend vers un point $P$ sur la variété $S_{t}$ le long d'un are $L$ situé à l'intérieur du domaine $\Omega_{t}$.

D'après les lemmes $1,2,3$ nous pouvons énoncer le théorème suivant: 
THÉORÈME 1. Si la densité $\varphi(Q, \tau)$ est une fonction continue et bornée sur la surface $s_{T}$, satisfaisant aux conditions de Liapounoff, la derivée transversale (13) du potentiel de simple couche au point intêrieur $X$ a domaine $\Omega_{t}$ tend uniformément ver's la limite

$$
\lim _{X \rightarrow P} \frac{d U(X, t)}{d T_{P_{t}}}=V(P, t)-\frac{(2 \sqrt{\pi})^{n} \varphi(P, t)}{2 \sqrt{\operatorname{det}\left[a^{i j}(P, t)\right]}}
$$

si le point $X$ tend vers le point $P$ de la variété $S_{\ell}$ pour $t>0$.

Nous avons encore les propriétés suivantes:

THÉORÈme 2. Si la densité $\varphi(Q, \tau)$ est une fonction bornée et intégrable sur la surface $s_{T}$, satisfaisant aux conditions de Liapounoff, le potentiel (3) de simple couche véritie, par rapport aux variables spatiales dans le domaine fer'mé $D_{T}$, la condition de Hölder:

$$
|U(X, t)-U(\bar{X}, t)| \leqslant \text { const } M_{p}|X \bar{X}|^{\vartheta},
$$

où l'exposant $\vartheta$ est un nombre positif arbitraire inférieur à l'unité.

Démonstration. Décomposons le potentiel (3) de simple couche en deux parties:

$$
\text { (47) } \begin{aligned}
U(X, t)= & \int_{0}^{t-\delta} \int_{S_{\tau}} \int_{\tau} \Gamma(X, t ; Q, \tau) \frac{\varphi(Q, \tau)}{\sin \left(N_{Q_{\tau}}, t\right)} d S_{Q} d \tau+ \\
& +\int_{t=\delta}^{t} \int_{S_{\tau}} \int \Gamma(X, t ; Q, \tau) \frac{\varphi(Q, \tau)}{\sin \left(N_{Q_{\tau}}, t\right)} d S_{Q} d \tau,
\end{aligned}
$$

où $\delta$ est un nombre positif assez petit pour que les inégalités (24) soient vraies, si $t-\tau<\delta$.

Le premier terme de la somme (47) est indéfiniment dérivable dans le domaine fermé $D_{T}$, donc le premier terme vérifie la contidion de Lipschitz dans ce domaine.

On peút ámontrer, comme le fait W. Pogorzelski (voir [2], démonstration du théorème 2), que le second terme de la somme (47) véritio la condition de Hölder de la forme (46).

En suivant aussi W. Pogorzelski (voir [2], démonstrations des théorèmes 3 et 4$)$, on démontre encore les théorèmes suivants:

THÉorème 3. Si la densité $p(Q, \tau)$ est une fonotion bornée et intégrable sur la surface $s_{T}$, satisfaisant aux conditions de Liapounoff, le potentiel (3) de simple couche vérifie, par rapport à la variable $t$ dans le domaine fermé $D_{1}$, la condition de Hölder:

$$
|U(X, t)-U(X, \bar{t})| \leqslant \text { const } M_{p}|t-\bar{t}|^{\vartheta / 2},
$$

oü l'exposant $\vartheta$ est un nombre positif arbitraire inférieur à l'unité.
ThÉoRł̀me 4. Si la densité $\varphi(Q, \tau)$ est une fonction bornée et intégrable sur la surface $s_{T}$, satisfaisant aux conditions de Liapounoff, l'intégrale $V(P, t)$ est une fonction définie sur la surface $s_{T}$, qui vérifie la condition ile Hölder :

$$
|\boldsymbol{V}(P, t)-V(\bar{P}, \bar{t})| \leqslant \text { const } M_{p}\left(|P \bar{P}|^{v a_{0}}+|t-\bar{t}|^{\vartheta^{\prime} a_{0} / 3}\right)
$$

où $a_{0}=\min \left(\alpha, \beta, 2 \beta^{\prime}\right)$, $\vartheta$ et $\vartheta^{\prime}$ sont des nombres positifs arbitraires inférteurs à l'unité.

\section{Travaux cités}

[1] W: Pogorzolski, Étude de la solution fondamentale de l'équation parabo. lique, Ricorehe di Matomatica 5, Napoli 1956, p. 25-57.

[2] - Propriétés des intégrales de l'équation parabolique normale, Ann. Polon. Math. 4 (1957), p. $61 \cdot 92$.

[3] M. Krzyżański, Równania różniezloowe czqstkowe ræędu drugiego I, Warszawa 1957.

Regu par la Rédaction le 27. 3. 1959 